\title{
Sizing Curve, Absorption Coefficient, Surface Chemistry, and Aliphatic Chain Structure of PbTe Nanocrystals
}

\author{
Joep L. Peters, ${ }^{\circledR}$ Jur de Wit, and Daniël Vanmaekelbergh** \\ Condensed Matter and Interfaces, Debye Institute for Nanomaterials Science, Utrecht University, P.O. Box 80000, 3508 TA \\ Utrecht, The Netherlands
}

\section{Supporting Information}

ABSTRACT: For colloidal semiconductor nanocrystals (NCs), the knowledge of the chemical structure and the size-dependent optical properties is of crucial importance, both from a practical and fundamental perspective. Here, we report the basic properties of $\mathrm{PbTe} \mathrm{NCs}$ in order to complement the already existing knowledge on $\mathrm{PbS}$ and $\mathrm{PbSe} \mathrm{NCs}$. The band gap versus $\mathrm{NC}$ diameter (sizing) curve was determined by combining transmission electron microscopy with absorption spectroscopy; the energy of the primary optical absorption follows $1 / d$ dependence with the diameter. The lead content of the NCs was determined with inductive coupled plasma optical emission spectrometry and the relative tellurium content with energy-dispersive
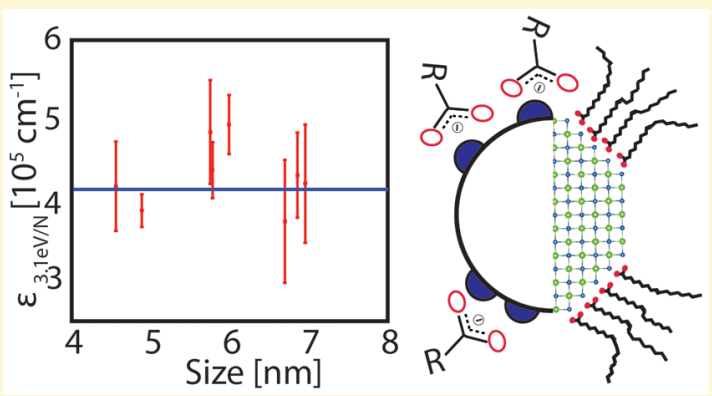
$\mathrm{X}$-ray spectroscopy. Combining these results yields a relation for the intrinsic absorption coefficient, which is independent of the $\mathrm{NC}$ size at $3.1 \mathrm{eV}$. The PbTe NCs are stabilized by $\mathrm{Pb}$ (oleate) $)_{2}$, but different from $\mathrm{PbS}$ NCs, oleate is predominantly bound in a chelating bidentate coordination. Besides that, we analyzed the structure of the aliphatic chains on all lead chalcogenide NCs and showed that the aliphatic chains are partly crystalline near the core and more liquid-like at the solvent side.

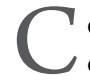
olloidal semiconductor nanocrystals (NCs) exhibit sizedependent optoelectronic properties, of interest, for a whole range of applications such as light-emitting devices, ${ }^{1}$ field-effect transistors, ${ }^{2-4}$ biomedical imaging, ${ }^{5-7}$ and photodetectors. $^{8,9} \mathrm{PbX}(\mathrm{X}=\mathrm{S}, \mathrm{Se}, \mathrm{Te}) \mathrm{NCs}$ are of special interest due to their tunable near-IR emission and large exciton Bohr radii. $^{10,11}$ Besides this, PbX NCs have been used as building blocks for several superstructures made by NC assembly, followed by oriented attachment. ${ }^{3,12-14} \mathrm{PbX}$ NCs have a rock salt crystal structure; attachment occurs exclusively via (100) facets on which the oleate capping is less strongly bound. ${ }^{15}$ Two-dimensional superstructures with several periodic motifs such as the square and honeycomb have been obtained; interestingly, they can all be considered as $2 \mathrm{D}$ plane cutouts from a simple cubic lattice. ${ }^{14,16}$

Because of the low effective mass of the electrons in $\mathrm{PbTe}$, the quantum coupling between attached NCs is even stronger than for the cases of $\mathrm{PbS}$ and $\mathrm{PbSe} .{ }^{17}$ For superstructures of $\mathrm{PbTe}$, novel electronic band structures can be expected, especially for the honeycomb geometry. Furthermore, because of the large mass of $\mathrm{Pb}$ and $\mathrm{Te}$, the spin-orbit coupling is strong; $\mathrm{PbTe}$ superstructures thus hold promise for future spintronics. ${ }^{18}$ In addition, once a PbTe superstructure is fabricated, the $\mathrm{Pb}^{2+}$ ion can be exchanged for $\mathrm{Cd}^{2+}$ and possibly $\mathrm{Hg}^{2+} \cdot{ }^{15}$ This can result in two-dimensional zinc blende geometric materials with exciting optoelectronics. For example; honeycomb superstructures composed of $\mathrm{HgTe}$ are predicted to show the quantum spin Hall effect almost up to room temperature. ${ }^{19} \mathrm{PbTe}$ is also particularly promising for energy conversion in high-ZT thermoelectric devices. ${ }^{20-23}$
Control of the nanogeometry is of crucial importance for these thermoelectric devices because the efficiency can be improved by promoting phonon scattering at crystal interfaces or nanoinclusions. $^{24,25}$ Hence, superstructures of PbTe NCs, fabricated in a bottom-up approach, can possibly result in cheap and well-defined materials for thermoelectrics.

Despite these prospects, colloidal PbTe NCs have been far less studied than the PbS and PbSe NC systems. This is due to the problematic chemistry, such as the low synthetic yield and extreme vulnerability to oxygen. Proper chemical synthesis and surface chemistry remains challenging even under the quasiinert conditions in a glovebox. ${ }^{26}$ Other difficulties are the discrepancy in the relation between the absorption peak and the NC size $\mathrm{s}^{2,27}$ and the absence of a way to determine the NC concentration. Furthermore, it has never been examined how (oleate) ligands bind to the PbTe facets, and how the ligand layer is structured; these topics are important to understand issues such as chemical (in)stability, shell-epitaxy, and $\mathrm{PbTe}$ NC self-assembly.

Here, we present experiments that resulted in the relation between the energy of the primary absorption peak and the $\mathrm{PbTe} \mathrm{NC}$ size. An empirical fit establishes a sizing curve, which follows $1 / d$ dependence. With the use of the Lambert-Beer law, we were able to determine the molar absorption coefficient at the band gap and at $3.1 \mathrm{eV}$. The intrinsic

Received: December 5, 2018

Revised: February 8, 2019

Published: February 8, 2019 
absorption coefficient at $3.1 \mathrm{eV}$ is size independent; the optical matrix element is entirely determined by the $\mathrm{PbTe}$ unit cells and crystal structure. We also investigated the surface chemistry with proton nuclear magnetic resonance $\left({ }^{1} \mathrm{H}\right.$ NMR) and Fourier transform infrared spectroscopy (FT-IR), and compared this with the other PbX NCs. This shows that all $\mathrm{PbX}$ are bound by z-type $\mathrm{Pb}$ (oleate) ${ }_{2}$ ligands. Because of the larger unit cell, the oleate is predominantly bound in a chelating bidentate coordination in PbTe NCs instead of a bridging bidentate coordination for $\mathrm{PbS}$. We also measured the structure of the oleate ligands on suspended $\mathrm{PbX} \mathrm{NCs} \mathrm{and}$ showed that the aliphatic chains are partly crystalline near the NC core, while the liquid-like part sticks into the solvent.

\section{THE PBTE SIZING CURVE}

The hot-injection method for the synthesis of PbTe NCs is developed by Murphy et al. ${ }^{27}$ and Urban et al. ${ }^{2}$ and used here to synthesize PbTe NCs in a diameter range between 3.5 and 7 $\mathrm{nm}$. Control over the NC size was most easily achieved by varying the oleic acid/ $\mathrm{Pb}$ ratio in the $\mathrm{Pb}$ precursor or by changing the growth time after injection. Both a higher oleic $\mathrm{acid} / \mathrm{Pb}$ ratio and a longer growth time resulted in larger NCs. The high-resolution transmission electron microscopy (HRTEM) images in Figure 1 show that the quasispherical PbTe
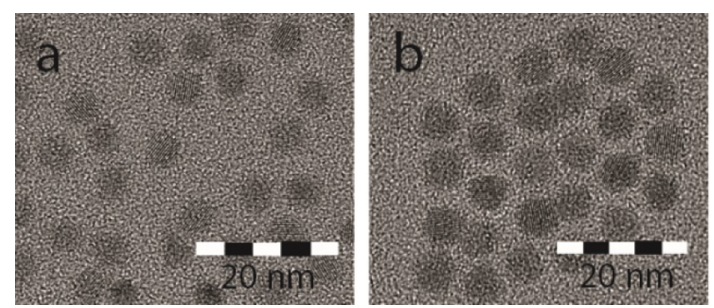

Figure 1. Two HR-TEM images of PbTe NCs of (a) 4.8 and (b) 6.2 $\mathrm{nm}$.

NCs are single-crystalline, without an amorphous layer on the $\mathrm{NC}$ surface. To determine the average size of the PbTe NCs in a given batch, approximately 300 particles per batch were analyzed in the same manner for each batch.

Four examples of absorption spectra of the NCs are plotted in Figure 2a, normalized at $400 \mathrm{~nm}$. Although the size dispersion is $<10 \%$, the band-edge peak overlaps with other transitions due to its complex band structure. This gives rise to a number of allowed transitions immediately after the first
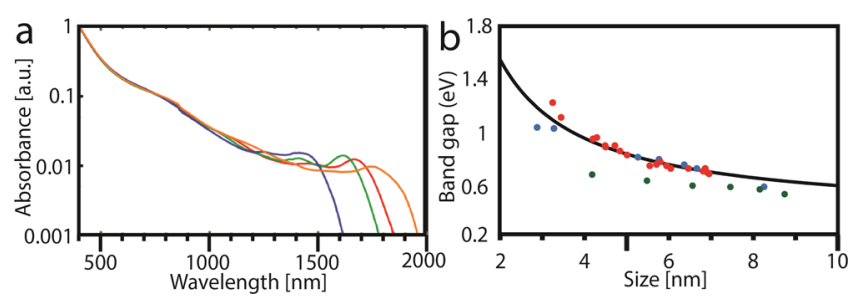

Figure 2. Absorption spectra and the size dependence of the band gap of PbTe NCs. (a) In the logarithmic graph, four absorption spectra of PbTe NCs dissolved in $\mathrm{C}_{2} \mathrm{Cl}_{4}$ are plotted and normalized at $400 \mathrm{~nm}$. The color coding relates to $\mathrm{PbTe} \mathrm{NCs}$ with the following sizes: blue $4.9 \mathrm{~nm}$, green $5.7 \mathrm{~nm}$, red $6.0 \mathrm{~nm}$, and orange $6.7 \mathrm{~nm}$. (b) Energy of the first maximum in the absorption, that is, the optical band gap, from this work (red) and previous work, that is, Murphy et al. ${ }^{27}$ (blue) and Urban et al. (green). ${ }^{2}$ exciton peak. ${ }^{27}$ Combining the peak position of the band edge with the NC size determined by transmission electron microscopy (TEM), we could establish a sizing curve as shown in Figure $2 \mathrm{~b}$. The red dots are PbTe NCs from this work, while the green dots are from Urban et al. ${ }^{2}$ and the blue dots are of Murphy et al. ${ }^{27}$ This shows that the data of Urban et al. deviate significantly from the other experimental data. We therefore excluded those data points in the fit for the sizing curve (black line).

The fitted sizing curve for $\mathrm{PbTe}$ is

$$
E_{0}=0.376+\frac{1}{0.42 d}
$$

Here, $E_{0}$ is the band gap in $\mathrm{eV}$, and $d$ is the NC diameter in $\mathrm{nm}$. The sizing curve allows us to determine the size of the PbTe NCs immediately from an absorption spectrum, instead of a tedious TEM study. An important factor in this equation is the constant 0.376 , which is close to the bulk band gap value of PbTe. ${ }^{25,28}$ Most sizing curves also have a $1 / d^{2}$ term, but its coefficient was too small to contribute in our equation. The predominance of the $1 / d$ term was also found experimentally and by calculation for $\mathrm{PbSe}$ and $\mathrm{PbS} \mathrm{NCs}^{29,30}$ The $1 / d$ dependence can be due to a combination of different effects, for example, exciton wave function spill-over, electron-hole many-body effects, and/or deviation from perfect parabolic bands. ${ }^{31}$ The same line of thought can be applied to PbTe, which has a more anisotropic band structure but does have a derivation from parabolicity near the band structure near the band edge. ${ }^{32}$ The contribution of the $1 / d^{2}$ term might become relevant if a larger NC size range is included. The current synthesis is however not suitable for very small or large NCs. Small NCs are often polydispersive which hinders the size determination, while for larger NCs, a shape change from spherical to quasicubical occurs, which broadens the absorption peak.

\section{QUANTIFYING LIGHT ABSORPTION}

We used inductive coupled plasma optical emission spectrometry (ICP-OES) to determine the $\mathrm{Pb}$ content in the PbTe NC suspensions investigated. The $\mathrm{Pb}^{2+}$ concentrations and their relative standard deviations (RSDs) are shown in Table S1. The sample purity was checked with ${ }^{1} \mathrm{H}$ NMR (see below). We were unable to reliably measure the $\mathrm{Te}^{2-}$ concentration with ICP-OES due to the formation of volatile hydrides. Therefore, we measured the atomic $\mathrm{Pb} / \mathrm{Te}$ ratios by energydispersive X-ray spectroscopy (EDX) shown in Table 1. The total amount of atoms per $\mathrm{NC}$ is calculated using eq 2, assuming a spherical shape.

$$
N=\frac{4 \pi}{3}(d / a)^{3}
$$

Table 1. Atomic Ratio and Total Number of Atoms per NC for Some PbTe NCs ${ }^{a}$

$\begin{array}{ccccc}\begin{array}{c}\mathrm{NC} \text { size } \\ (\mathrm{nm})\end{array} & \begin{array}{c}\mathrm{Pb} \text { atomic } \\ (\%)\end{array} & \begin{array}{c}\mathrm{Te} \text { atomic } \\ (\%)\end{array} & \begin{array}{c}\mathrm{Pb} / \mathrm{Te} \\ \text { ratio }\end{array} & N \text { (atoms/NC) } \\ 4.87 & 53.87(0.15) & 46.13(0.10) & 1.16 & 1827 \\ 5.74 & 55.24(0.42) & 44.75(0.31) & 1.24 & 2992 \\ 5.98 & 54.09(0.49) & 45.90(0.30) & 1.18 & 3383 \\ 6.85 & 53.59(1.65) & 46.40(0.95) & 1.15 & 5085\end{array}$

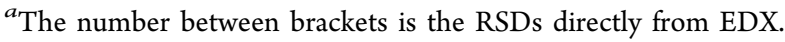


where $a$ is the lattice parameter, and $d$ is the particle diameter, both in $\mathrm{nm} .{ }^{33}$ The amount of atoms is corrected for the uneven $\mathrm{Pb} / \mathrm{Te}$ ratio, $R$ in eq 3 . The number of NCs then follows from the total amount of $\mathrm{PbTe}$ unit cells measured in the sample divided by the number of unit cells per NC. Or in other words, the $\mathrm{NC}$ concentration $C_{\mathrm{NC}}$ is given by

$$
C_{\mathrm{NC}}=\frac{1}{N} \frac{(1+R)}{R} C_{\mathrm{m}}
$$

Here, $C_{\mathrm{m}}$ is the lead concentration in the sample. We measured an absorption spectrum and combined it with the $\mathrm{PbTe} \mathrm{NC}$ concentrations to calculate the molar absorption coefficient (also known as molar extinction coefficient) at the band gap, and at $3.1 \mathrm{eV}$.

\section{ABSORPTION COEFFICIENT AT BAND GAP}

The band-edge absorption peak is fitted with Gaussian and integrated to get a measure of the $\mathrm{NC}$ absorbance of a given sample. From this, the energy-integrated molar absorption coefficient at the band gap $\varepsilon_{\text {gap }}$ is derived using the LambertBeer law

$$
\log \frac{I}{I_{0}}=-\varepsilon c l=-A
$$

where $A$ is the absorbance or absorptivity of our suspension for an optical path length $l$. $A=$ dimensionless, $\varepsilon=$ the molar absorption coefficient $\left(\mathrm{mol}^{-1} \mathrm{~cm}^{-1}\right)$, and $c$ denotes the molar concentration of PbTe NCs. Figure 3a presents an overview of
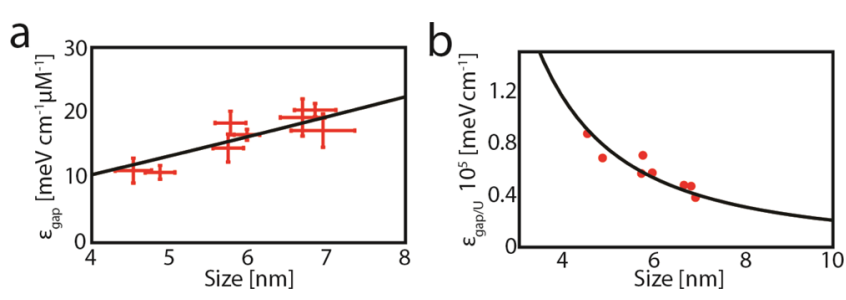

Figure 3. Absorption coefficient of PbTe NCs at the band gap. (a) shows the relation between the energy-integrated molar absorption coefficient at the band gap to the NC size. The horizontal errors account for one standard deviation for the NC size determined by TEM image analysis. The vertical error bar originates from EDX and ICP-OES measurements. (b) Intrinsic energy-integrated absorption coefficient $\varepsilon_{\text {gap } / \mathrm{U}}$ to the NC size is plotted with an inverse quadratic relation.

the experimental data relating energy-integrated $\varepsilon_{\text {gap }}$ ( $\mathrm{meV}$ $\mathrm{mol}^{-1} \mathrm{~cm}^{-1}$ ) to NC size. Similar as for PbSe and $\mathrm{PbS},{ }^{30}$ a power $\log$ is used to fit the data. This resulted in the following relation

$$
\varepsilon_{\text {gap }}=2.2 d^{1.1}
$$

The relation obtained from the data is similar compared to the energy-integrated molar absorption coefficients of $\mathrm{PbS}$ and PbSe. ${ }^{29,30}$ PbTe NCs, however, absorb less light at the band gap compared to other lead chalcogenides. An additional means of comparison is the energy-integrated absorption coefficient $\varepsilon_{\text {gap } / U}$, per unit cell (U), which is calculated with the following formula, ${ }^{30}$ and shown in Figure $3 \mathrm{~b}$

$$
\varepsilon_{\text {gap } / \mathrm{U}}=\frac{6 \ln (10) \varepsilon_{\text {gap }}}{\pi d^{3} N_{\mathrm{A}}}=15.5 d^{-1.9}
$$

This shows that $\varepsilon_{\text {gap } / U}$ is size dependent and increases with decreasing size.

\section{ABSORPTION COEFFICIENT AT 3.1 EV}

We also applied the Lambert-Beer law using the absorption at $3.1 \mathrm{eV}$. For other NCs, it was found that at this energy, far above the first optical transition, the effects of quantum confinement on the absorption coefficient per unit cell can be neglected. $^{29,30,33,34}$ This would mean that the absorbance per $\mathrm{NC}$ should be simply proportional to the number of $\mathrm{PbTe}$ unit cells, thus to the NC volume. The experimental results are presented in Figure 4a, which can be fitted with

$$
\varepsilon_{3.1 \mathrm{eV}}=(0.05846 \pm 0.002) d^{3}
$$
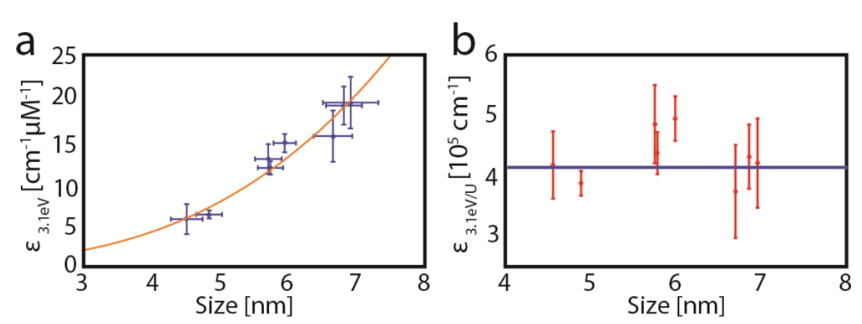

Figure 4. Absorption coefficient of PbTe NCs at $3.1 \mathrm{eV}$. (a) Molar absorption coefficient at $3.1 \mathrm{eV}$ for different sizes of PbTe NCs. The data points follow $d^{3}$ dependence. (b) Intrinsic absorption coefficient $\varepsilon_{3.1 \mathrm{eV} / \mathrm{U}}$ is size independent, as seen from the red dots. The blue line is the calculated $\varepsilon_{3.1 \mathrm{eV} / \mathrm{U}}$ using the bulk $\mathrm{PbTe}$ dielectric function.

$\varepsilon_{3 . \mathrm{eV}}\left(\mathrm{cm}^{-1} \mathrm{~mol}^{-1}\right)$ can be divided by Avogadro's constant, and the NC volume to yield the intrinsic absorption coefficient (i.e., per $\mathrm{PbTe}$ unit cell) is denoted as $\varepsilon_{3.1 \mathrm{eV} / \mathrm{U}}$. This intrinsic absorption coefficient at this energy (sometimes also symbolized by $\mu$ ) is independent of the NC size (Figure 4b), similar as for other systems. ${ }^{29,30,33,34}$ We note that the absorption coefficient per unit cell at the first transition decreases with increasing size to a value equal to the bulk value.

The size-independent absorption coefficient at $\varepsilon_{3.1 \mathrm{eV} / \mathrm{U}}$ can also be calculated, using the Maxwell-Garnett effective medium theory. ${ }^{33,35}$ For these calculations, the dielectric constant of the solvent tetrachloroethylene ${ }^{36}$ and the dielectric function of bulk $\mathrm{PbTe}$ are needed. The available literature for the dielectric function of bulk $\mathrm{PbTe}$ uses tight-binding calculations and band-structure approximations. ${ }^{32,37}$ The most reliable results for the dielectric function and absorption coefficient of $\mathrm{PbTe}$ are measured with spectroscopic ellipsometry, which directly measures both real and imaginary components of the dielectric function. ${ }^{38}$ Based on their data, the local field effect is calculated by the following equation

$$
f_{\mathrm{LF}}=\frac{3 \varepsilon_{\mathrm{s}}}{\left|\tilde{\varepsilon}_{\mathrm{PbTe}}+2 \varepsilon_{\mathrm{s}}\right|}
$$

where $\tilde{\varepsilon}$ is the composite dielectric function, and $\varepsilon_{\mathrm{s}}$ is the dielectric function of the solvent. The calculated intrinsic absorption coefficient can subsequently be calculated with

$$
\varepsilon_{3.1 \mathrm{eV} / \mathrm{U}}=\frac{2 \pi}{\lambda n_{\mathrm{s}}}\left|f_{\mathrm{LF}}\right|^{2} \varepsilon_{\mathrm{PbTe}, \mathrm{I}}=\frac{\ln (10) A}{f l}
$$

where $f$ is the volume fraction, $n_{\mathrm{s}}$ is the refractive index of the solvent, and $\varepsilon_{\mathrm{PbTe}, \mathrm{I}}$ is the imaginary part of the dielectric 
function of PbTe. The calculated value is added in Figure $4 \mathrm{~b}$ as a blue line. It shows that the calculated and measured intrinsic absorption coefficients are in good agreement.

\section{SURFACE CHEMISTRY OF PBTE NCS}

In the case of $\mathrm{PbSe}$ and $\mathrm{PbS} \mathrm{NCs}$, the ligands have been investigated with FT-IR and ${ }^{1} \mathrm{H}$ NMR. ${ }^{30,39,40}$ In Figure 5a, we

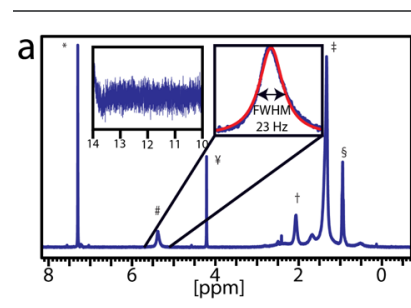

C

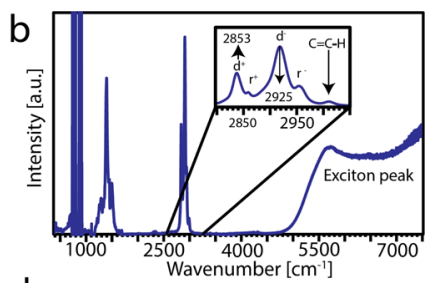

d
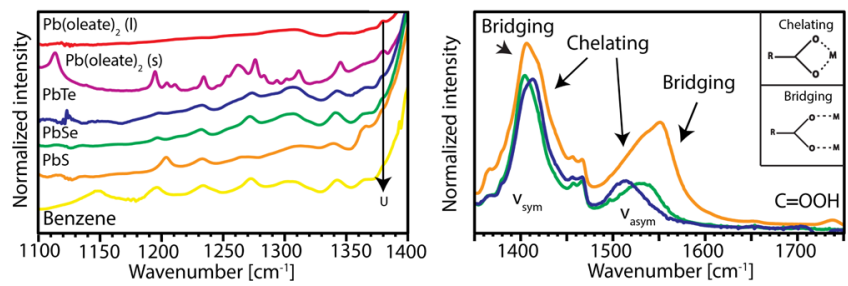

Figure 5. Surface chemistry of PbTe NCs. (a) shows a typical ${ }^{1} \mathrm{H}$ NMR spectrum of PbTe NCs dissolved in chloroform- $d$, with the left inset showing a zoomed-in image on the region of a possible acid peak and the right inset showing a fit of the vinyl peak, with a full width at half-maximum of $23 \mathrm{~Hz}$. The symbols stand for: * chloroform, \# vinyl peak, and $¥$ from ferrocene which is added in a known concentration as the internal standard, $\S$ methyl group, $\ddagger$, and $\dagger$ from other hydrogens in the aliphatic chain. The integral and position of the peaks identifies oleate bound on the NC surface. (b) FT-IR spectrum for $\mathrm{PbTe}$ NCs of $7.2 \mathrm{~nm}$ in diameter. The saturated bands around 800 and $900 \mathrm{~cm}^{-1}$ are from the tetrachloroethylene solvent. The inset shows the $\mathrm{CH}$ stretch region with $\mathrm{d}^{+}$and $\mathrm{d}^{-}$, the symmetric and antisymmetric methylene $-\mathrm{CH}_{2}-$ stretches, and $\mathrm{r}^{+}$and $\mathrm{r}^{-}$, symmetric and antisymmetric methyl $-\mathrm{CH}_{3}$ stretches. Also, note the clear presence of the $-\mathrm{C}=\mathrm{C}-\mathrm{H}$ stretch. (c) shows FT-IR magnified on the methylene wagging and twisting-rocking progression bands. The graphs are normalized on the $\mathrm{CH}_{2}$ stretch at $2925 \mathrm{~cm}^{-1}$. The red curve of dissolved $\mathrm{Pb}$ (oleate) $)_{2}$ shows no clear peaks, while clear peaks are visible for solid $\mathrm{Pb}$ (oleate $)_{2}$ in purple. The blue, green, orange, and yellow graphs are, respectively, $\mathrm{PbTe}, \mathrm{PbSe}, \mathrm{PbS}$ NCs dissolved in tetrachloroethylene (TCE), and $\mathrm{PbTe}$ NCs dissolved in benzene. The peaks indicated with $U$ are the methyl symmetric bending (umbrella mode) vibration. (d) shows the carboxylate stretch region, with the symmetric $\left(\nu_{\mathrm{s}}\right)$ and asymmetric carboxylate $\left(\nu_{\mathrm{as}}\right)$ stretch. The graphs are normalized on the $\mathrm{CH}_{2}$ stretch at $2925 \mathrm{~cm}^{-1}$. Note that the bands are broad and consist of doublets which are deconvoluted in Figure S2. The underlying peaks are indicative for the type of oleate coordination, either a chelating or bridging bidentate coordination. There is no peak visible at $1710 \mathrm{~cm}^{-1}$ showing the absence of oleic acid.

show a typical ${ }^{1} \mathrm{H}$ NMR spectrum for PbTe NCs dissolved in chloroform- $d$. There are no sharp peaks visible, besides the peaks originating from the solvent molecules, chloroform- $d$, and ferrocene (internal standard). This shows that all other moieties are bound to the NC surface and there are no unreacted precursors in the suspension. The ${ }^{1} \mathrm{H}$ NMR peak positions and integrals correspond to oleate on the NC surface, which is also confirmed by the absence of an acid peak (left inset Figure 5a).
A typical FT-IR spectrum of PbTe NCs is shown in Figure $5 \mathrm{~b}$ and also confirms the presence of oleates as major species on the $\mathrm{PbTe} \mathrm{NC}$ surface. The two saturated peaks around 800 and $900 \mathrm{~cm}^{-1}$ originate from the tetrachloroethylene solvent. The inset of Figure $5 \mathrm{~b}$ shows the $\mathrm{CH}$ stretch region, which matches with oleate ligands. ${ }^{39}$ There are no other broad or sharp peaks, which show the absence of hydroxide or water as a major component on the PbTe NC surfaces, similar as found for PbSe. ${ }^{39}$ Figure $5 \mathrm{~d}$ shows a higher magnification on the carboxylic region, where there is no acid peak visible around $1710 \mathrm{~cm}^{-1}$. This confirms that it is oleate that is bound to the NC surface, not oleic acid.

$\mathrm{PbTe}$ NCs are dissolved in an apolar solvent, so this would suggest that the negatively charged oleate needs to be charge compensated by positively charged lead atoms. This is confirmed by our EDX analysis in Table 1 showing an excess of $\mathrm{Pb}^{2+}$ ions compared to $\mathrm{Se}^{2-}$; the excess of $\mathrm{Pb}^{2+}$ ions on $\mathrm{PbTe} \mathrm{NCs}$ is similar to that reported for $\mathrm{PbS}$ and $\mathrm{PbSe}$ NCs. ${ }^{29,30}$ This strongly suggests that the PbTe NCs are capped by $\mathrm{Pb}$ (oleate $)_{2}$ as $\mathrm{z}$-type ligands, with oleate charge compensated by excess lead.

The ligand density of several samples was determined with either ${ }^{1} \mathrm{H}$ NMR by the use of an internal standard or FT-IR by the use of a calibration curve. ${ }^{39,41,42}$ This resulted in similar values as found for other semiconductor NCs, ${ }^{39,40}$ ranging from 2.2 to 4.6 oleate $/ \mathrm{nm}^{2}$.

\section{OLEATE COORDINATION}

The carboxylic vibrations for all three PbX NCs are shown in Figure 5d, normalized on the $\mathrm{CH}_{2}$ stretching vibration at 2925 $\mathrm{cm}^{-1}$. This shows remarkable differences in the intensities and positions of the symmetric $\left(\nu_{\text {sym }}\right)$ and asymmetric $\left(\nu_{\text {asym }}\right)$ stretch vibrations for the three types of $\mathrm{Pb}$-chalcogenide NCs. We want to remark that the ligand density can alter the carboxylic coordination, but the effects seen here are stronger. ${ }^{39}$ Also, the NC size and solvent does not influence the coordination significantly (Figures S1 and S2). This means that the results should be related to differences between $\mathrm{PbS}$, $\mathrm{PbSe}$, and PbTe cores.

The energy difference between the symmetric and asymmetric carboxylate stretch is often used to assign the carboxylic bonding coordination. ${ }^{43-45}$ There is however quite some discussion regarding the generality of this method. ${ }^{46}$ Recently, it has been shown by a combination of FT-IR and structural information that similar band positions, as displayed, in Figure 5d, can be attributed to bidentate bridging and chelating coordinations ${ }^{47,48}$ because a monodentate coordination has a higher $\Delta \nu .^{46}$ The broadening and non-Lorentzian shape of the bands shown here indicate that both types of carboxylic coordination are present on all $\mathrm{PbX}$ surfaces. In Figure S3 and Table S2, we deconvoluted $\nu_{\text {sym }}$ and $\nu_{\text {asym }}$ by fitting two Lorentzians under each $\nu_{\text {sym }}$ and $\nu_{\text {asym }}$ to separate the different components. Cass et al. found that the low wavelength peak of the $\nu_{\text {sym }}$ vibration and the high wavelength peak $\nu_{\text {asym }}$ belong to the bridging bidentate carboxylic coordination, ${ }^{43}$ while the high wavelength peak of $\nu_{\text {sym }}$ and the low wavelength peak of $\nu_{\text {asym }}$ belong to the chelating coordination (see Figure 5d). The assignment was, among other things, based on the observation that the intensity of the coupled peaks vary simultaneously. This seems also to be true for our PbX NCs; the chelating combination has a higher intensity for $\mathrm{PbTe}$ (and $\mathrm{PbSe} \mathrm{NCs}$ ), while the bridging peaks are more intense for $\mathrm{PbS}$. The energy difference also matches 
the assignment of the coordination because a chelating bidentate coordination generally has a lower $\Delta \nu$ than a bridging bidentate coordination. The energy difference for the chelating bidentate coordination of all three $\mathrm{PbX}$ ranges from 90 to $97 \mathrm{~cm}^{-1}$, while it ranges from 131 to $151 \mathrm{~cm}^{-1}$ for the bridging bidentate coordination. The intensities of the bands for $\mathrm{PbX} \mathrm{NCs}$ suggest that the amount of bridging coordinations decreases going from $\mathrm{S}$ and Se to Te. It should be noted that it is expected that there is some variations in the peak position and intensity of $\nu_{\text {sym }}$ and $\nu_{\text {asym }}$ for different $\mathrm{PbX}$ NCs due to differences in the lead carboxylate bonding angle and variation in the $\mathrm{Pb}-\mathrm{O}$ bond distance. ${ }^{44,47,49}$

The dominant chelating bidentate coordination for $\mathrm{PbTe}$ $\mathrm{NCs}$ can be reasoned by the larger unit cell size. The $\mathrm{Pb}$ density on the (111) facet of the rock salt PbX crystal, which binds most of the oleate ligands, ${ }^{39}$ is $6.55,6.15$, and $5.53 \mathrm{~nm}^{-2}$, for $\mathrm{PbS}, \mathrm{PbSe}$, and $\mathrm{PbTe}$, respectively. The lead atoms on the (111) facets are partly integrated into the crystal lattice, ${ }^{39}$ which means that one exposed lead atom on the (111) facet should be charge compensated by one oleate. The density of aliphatic chains is however sterically limited to approximately 4-4.6 chains $\mathrm{nm}^{-2}$ on planar surfaces. ${ }^{50,51}$ This means that in the case of $\mathrm{PbS}$, there are much more lead atoms compared to carboxylate groups, meaning that some lead atoms might "share" one carboxylate, resulting in a bridging coordination. Similarly, it was recently found that the $\mathrm{Pb}-\mathrm{Pb}$ distance in crystalline metal soaps is $4.45 \AA$. This crystal contained a combination of bridging and chelating bidentate coordinations. ${ }^{47}$ The $\mathrm{Pb}-\mathrm{Pb}$ distance on the (111) facets in $\mathrm{PbS}, \mathrm{PbSe}$, and $\mathrm{PbTe}$ is, respectively, 4.20, 4.33 and $4.57 \AA$, which shows that the likelihood for bridging coordinations is the highest for $\mathrm{PbS}$.

\section{ALIPHATIC CHAIN STRUCTURE IN SOLUTION}

FT-IR also provides structural information about the aliphatic part of the oleate ligands on the NC surface. The most important feature is the progression of bands between 1150 and $1400 \mathrm{~cm}^{-1}$, which shows a series of well-defined peaks for all-trans crystalline chains and can be assigned to the twistingrocking and wagging $\mathrm{CH}_{2}$ vibration. ${ }^{52,53}$ The $\mathrm{CH}_{2}$ groups in a liquid-like state can move freely, resulting in a large number of rotational isomers which smears the peaks out, and eventually even disappear for liquid chains. ${ }^{52,53}$ This is clearly visible when you compare dissolved $\mathrm{Pb}$ (oleate $)_{2}$ and solid $\mathrm{Pb}$ (oleate $)_{2}$ in Figure 5c. The only peak which is still recognizable in the dissolved $\mathrm{Pb}$ (oleate) $)_{2}$ spectrum is the umbrella mode of $\mathrm{CH}_{3}$ (indicated with a $\mathrm{U}$ ), which does not depend on chain packing.

The progression bands of solid $\mathrm{Pb}$ (oleate) $)_{2}$ show much resemblance with progression bands recently resolved for the crystalline aliphatic chain of $\mathrm{Zn}$ (oleate) $)_{2} .{ }^{54}$ These authors showed that in comparison with $\mathrm{Zn}$ (stearate) $)_{2}$, the hydrocarbon chain before and after the double bond melts independently and therefore has a rather complicated peak pattern. ${ }^{54}$ Recently, Martinez-Casado et al. identified all phase transitions of several saturated lead(II) soaps. ${ }^{47}$ The hydrocarbon chain packing changes from a completely crystalline phase to a rotator phase in which the alkyl chains have rotational freedom. The phase can change further to a liquid crystal before it completely melts to a stable isotropic liquid. ${ }^{47}$ Research on gold and silver NCs showed that the saturated aliphatic chains are partly crystalline; the chains pack in an alltrans conformation near the NC core, while the chains are more liquid-like at the end of the chain. ${ }^{55-60}$ Another indication of a partially crystalline phase is the exact peak position of the $\mathrm{CH}_{2}$ stretch vibration. The exact peak positions of the symmetric and asymmetric $\mathrm{CH}_{2}$ stretch vibration for the crystalline chain are 2850 and $2920 \mathrm{~cm}^{-1}$, respectively. Because of the presence of gauche disorder in the alkyl chain, these peaks shift to higher frequencies for the rotator phase, namely, 2856 and $2928 \mathrm{~cm}^{-1}$. $^{47}$ We found a frequency in the range of 2852-2854 and 2924-2926 $\mathrm{cm}^{-1}$ for PbX NCs larger than approximately $4 \mathrm{~nm}$. More freedom of movement at the end of the chain can be seen from the absence of peak splitting of the methyl $\left(\mathrm{CH}_{3}\right)$ group at $2957 \mathrm{~cm}^{-1}\left(\mathrm{r}^{-}\right.$, inset Figure $\left.5 \mathrm{~b}\right)$. This points to aliphatic chains that are neither fully crystalline nor fully liquid-like. Because of the different melting behaviors of the aliphatic chain before and after the double bond of oleate, ${ }^{54}$ it is tempting to suggest a predominantly liquid-like phase outward from the double bond to the methyl group and a more crystalline phase inward from the double bond to the NC core.

\section{ALIPHATIC CHAIN STRUCTURE IN DIFFERENT SOLVENTS}

NCs which are dissolved in benzene show an even more pronounced sequence of peaks, indicating a more solid-like ligand shell (see Figure 5c). The energy difference between the six peaks is approximately equal, suggesting that the complete chain from the core to the double bond is in a solid-like state. Recently, it was found that a broader peak in ${ }^{1} \mathrm{H}$ NMR indicates less solvated ligands. ${ }^{61}$ The peak width of $5.1 \mathrm{~nm}$ $\mathrm{PbSe} \mathrm{NCs}$ dissolved in different solvents confirms this trend. The ${ }^{1} \mathrm{H}$ NMR spectra are shown in Figure S4, and a comparison of the vinyl peak width is shown in Table 2 .

Table 2. Peak Width of the Vinyl Peak in the ${ }^{1} \mathbf{H}$ NMR Spectra of Oleate-Capped $5.1 \mathrm{~nm}$ PbSe NCs

$\begin{array}{lc} & \text { vinyl peak width }[\mathrm{Hz}] \\ \text { chloroform- } d & 23 \\ \text { hexane- } d_{14} & 30 \\ \text { benzene- } d_{6} & 54 \\ \text { toluene- } d_{8} & 56 \\ \text { chlorobenzene- } d_{5} & 47\end{array}$

There is more broadening of the peaks when NCs are dissolved in an aromatic solvent, compared to the smaller tetrachloroethylene and hexane. This indicates that the aromatic solvent molecules are too large to penetrate deeply into the ligand chain, which results in a more solid-like ligand shell. Similar as found before, also halocarbons seem to penetrate deeper into the ligand corona compared to hydrocarbons. ${ }^{62}$

These findings not only contribute to a better understanding of ligand-ligand interaction in a capping layer but also of the interaction between NCs during self-assembly. Recently, it was shown that these interactions have a large influence on the NC nucleation and growth, ${ }^{63}$ solubility, ${ }^{64,65}$ and self-assembly. ${ }^{62,66-68}$

\section{CONCLUSIONS}

Unlike for $\mathrm{PbS}$ and $\mathrm{PbSe} \mathrm{NCs}$, a number of basic properties of $\mathrm{PbTe} \mathrm{NCs}$ were not investigated so far. Above, we reported some crucial optical, chemical, and structural characteristics of state-of-the-art PbTe NCs. First, we established the size dependency of the band gap, demonstrating strong quantum 
confinement. We also determined the relation between an absorption spectrum and the PbTe NC concentration. This was done by using the integrated absorbance of the band-edge peak and the absorption at $3.1 \mathrm{eV}$. The absorbance per PbTe unit cell is size independent at high energy and equal to the bulk value. Next, we compared the surface chemistry of all three types of $\mathrm{Pb}$-chalcogenide NCs. This shows that for common synthesis procedures of $\mathrm{PbX} \mathrm{NCs,} \mathrm{Pb}$ (oleate) $)_{2}$ is the primary z-type ligand. The binding coordination of the oleate is a combination of bridging and chelating bidentate coordination. Because of limited space on the NCs surface, $\mathrm{PbS}$ has the most bridging configurations, while $\mathrm{PbTe}$ has the least. Last, we also address the aliphatic chain structure of these oleate ligands on the $\mathrm{PbX}$ NCs. This shows that part of the ligand shell closest to the surface is crystalline, while further away from the surface, the ligands are organized in a more liquid-like fashion.

\section{EXPERIMENTAL SECTION}

Chemicals. 1-Butanol (anhydrous) (99.8\%), 1-octadecene (90\%), acetonitrile (anhydrous) (99.8\%), cadmium(II) acetate dihydrate ( $\geq 99.99 \%)$, cadmium(II) oxide ( $\geq 99.99 \%)$, D-chloroform (99.8\%), diphenyl ether (99\%), ethanol (anhydrous) (99.8\%), hexane (anhydrous) (95\%), lead acetate trihydrate $(\geq 99.99 \%)$, methanol (anhydrous) (99.8\%), oleic acid (90\%), tellurium (325 or 200 mesh) (99.99\%), tetrachloroethylene (99.999\%), toluene (anhydrous) (99.8\%), and trioctylphosphine (TOP; 97\%), were all purchased from Sigma-Aldrich and used without purification.

$\mathrm{PbTe}$ synthesis was performed based on the method of Urban et $\mathrm{al}^{2}$ The $\mathrm{Pb}$ precursor was synthesized in a fumehood by the addition of $1.138 \mathrm{~g}$ lead acetate trihydrate $\left(\mathrm{Pb}(\mathrm{Ac})_{2} \cdot 3 \mathrm{H}_{2} \mathrm{O}\right), 3.3 \mathrm{~mL}$ of oleic acid, and $8 \mathrm{~mL}$ of octadecene in a three-neck flask. The three-neck flask was put under vacuum in a Schlenk line and flushed with nitrogen $\left(\mathrm{N}_{2}\right)$ in order to get rid of $\mathrm{O}_{2}$ in the reaction atmosphere. This cycle was repeated at least three times. After flushing, the mixture was put under vacuum again, and the temperature was slowly increased to $130{ }^{\circ} \mathrm{C}$. Solid $\mathrm{Pb}(\mathrm{Ac})_{2} \cdot 3 \mathrm{H}_{2} \mathrm{O}$ reacts with oleic acid resulting in the evaporation of acetate and water. The temperature was maintained at $130{ }^{\circ} \mathrm{C}$ for at least $3 \mathrm{~h}$, until no bubbling was visible anymore. The tellurium precursor was completely prepared in the glovebox because of its sensitivity to oxygen. Tellurium (0.194 g) was combined with $3 \mathrm{~mL}$ of TOP and stirred at $\sim 90{ }^{\circ} \mathrm{C}$, until the solution was completely transparent and yellow.

The previously prepared $\mathrm{Pb}$-oleate is first heated to $170{ }^{\circ} \mathrm{C}$ in a three-necked flask in a Schlenk line, after which TOP-Te is rapidly injected under vigorous stirring. Upon injection, the solution immediately turns black. It is of key importance that the injection happens quickly and without stopping the stirring, in order to avoid concentration gradients in the mixture. After $4 \mathrm{~min}$, the growth process was quenched by quickly injecting $3 \mathrm{~mL}$ of hexane into the solution. Further cooling of the reaction was achieved by placing the flask in a water bath or blowing cool air onto the flask. Different sizes can be formed through varying reaction times, injection temperature, and oleic acid/lead ratio.

Once the solution had cooled down to room temperature, the $\mathrm{PbTe}$ NCs were washed inside a glovebox. The first step was to add water free ethanol with an approximate ratio of $2 / 3$ to the original volume. If the system turned from oily black to dark gray and flaky, it was sufficiently destabilized and placed in a centrifuge. The precipitate was then separated from the supernatant and redispersed in hexane. This washing cycle was repeated five times for every sample in order to get rid of unwanted reaction products and leftover precursors.

The $\mathrm{PbTe} \mathrm{NC}$ synthesis was performed under inert conditions in a glovebox, and all other experiments were performed as much as possible under inert conditions. Although a slight oxidation of the $\mathrm{PbTe}$ surface cannot be excluded, ${ }^{26}$ we could not find indications of this in the TEM images and in our large data set on the surface chemistry.
The solid lead oleate was made via the method of Hendricks et al. ${ }^{69}$ In short, lead(II) oxide (10.00 g, $44.8 \mathrm{mmol})$ and acetonitrile ( 20 $\mathrm{mL}$ ) are added to a $100 \mathrm{~mL}$ round-bottom flask. The suspension was stirred while being cooled in an ice bath, after which trifluoroacetic acid $(0.7 \mathrm{~mL}, 8.96 \mathrm{mmol}, 0.2$ equiv) and trifluoroacetic anhydride $(6.2 \mathrm{~mL}, 44.8 \mathrm{mmol}, 1$ equiv) were added. After $10 \mathrm{~min}$, the yellow lead oxide dissolved, resulting in a clear and colorless solution that was allowed to warm to room temperature. Oleic acid $(25.437 \mathrm{~g}$, $90.05 \mathrm{mmol}, 2.01$ equiv), isopropanol $(\sim 180 \mathrm{~mL})$, and triethylamine $(10.246 \mathrm{~g}, 101.25 \mathrm{mmol}, 2.26$ equiv) were added in a $500 \mathrm{~mL}$ Erlenmeyer flask. The lead trifluoroacetate solution was slowly added to the oleic acid solution while stirring, resulting in the formation of a white precipitate. The mixture was heated to reflux and dissolve the precipitate, after which a clear and colorless solution was obtained. The heat was turned off, and the flask was allowed to cool to room temperature, followed by further cooling in a $-20^{\circ} \mathrm{C}$ freezer for $>2 \mathrm{~h}$. The resulting white powder was isolated by suction filtration using a glass-fritted funnel, and the filtrate was thoroughly washed with methanol $(3 \times 300 \mathrm{~mL})$. Large pieces were crushed to get a white powder. This was subsequently dried under vacuum for $>6 \mathrm{~h}$ to get a fluffy white powder.

$\mathrm{PbSe} \mathrm{NCs}$ used for the comparison were made using a procedure described by Campos et al. ${ }^{70}$ The solid Pb-oleate was used as a $\mathrm{Pb}$ precursor, while the selenium precursor ( $N$-cyclohexylpyrrolidine-1carboselenoamide) was prepared by mixing selenium $(9.0 \mathrm{mmol})$, pyrrolidine $(9.0 \mathrm{mmol})$, cyclohexyl isocyanide $(9.0 \mathrm{mmol})$, and $10 \mathrm{~mL}$ toluene. This mixture was heated to $\sim 100{ }^{\circ} \mathrm{C}$ until a clear solution was obtained. If black solid selenium was still present, additional pyrrolidine and/or cyclohexyl isocyanide was added to make sure that all selenium reacted. The mixture was allowed to cool to room temperature at which the selenourea precipitated. The liquid was decanted, and the obtained white solid was placed under vacuum for $24 \mathrm{~h}$. Subsequently, the solid was redissolved in $10 \mathrm{~mL}$ toluene at 100 ${ }^{\circ} \mathrm{C}$ and allowed to cool down. The precipitate was filtered, and the solid was placed under vacuum for another $24 \mathrm{~h}$.

The synthesis of PbSe was performed in a Schlenk line where $1.6 \mathrm{~g}$ (2.08 mmol, 1.2 equiv) $\mathrm{Pb}(\text { oleate })_{2}$ was dissolved in $140 \mathrm{~mL}$ hexadecane in a $250 \mathrm{~mL}$ three-neck flask. $0.45 \mathrm{~g}(1.7 \mathrm{mmol}, 1$ equiv $)$ $N$-cyclohexylpyrrolidine-1-carboselenoamide was dissolved in $8 \mathrm{~mL}$ diphenyl ether. Both precursors were heated to $100{ }^{\circ} \mathrm{C}$ to yield a clear colorless solution. The selenourea was quickly injected into the $\mathrm{Pb}$ (oleate $)_{2}$ solution which turned brown in approximately $8 \mathrm{~s}$. After $110 \mathrm{~min}$, the reaction was cooled down with an ice bath and inserted inside the glovebox. $90 \mathrm{~mL}$ of $n$-butanol and $60 \mathrm{~mL}$ of methanol was added to precipitate the NCs. The mixture was centrifuged, and the black residue was dissolved in $10 \mathrm{~mL}$ toluene. This solution was washed three more times with methanol.

Characterization. UV-Vis. A PerkinElmer LAMBDA $950 \mathrm{UV} / \mathrm{vis}$ spectrometer was used to conduct absorption measurements in the wavelength range from 300 to $2300 \mathrm{~nm}$. The samples were prepared in the glovebox by drying $5-30 \mu \mathrm{L}$ of $\mathrm{PbTe}$ solution under vacuum. The residue was then redispersed in $3 \mathrm{~mL}$ TCE because of the absence of absorption features in the near-infrared.

The information captured in the absorption spectrum was acquired in two different ways. First, in the $300-400 \mathrm{~nm}$ wavelength region, the volume-corrected absorption was used for further calculations. The second region is the first exciton peak and is different per sample in terms of the peak position and width.

Fourier Transform Infrared Spectroscopy. FT-IR measurements were performed in Bruker vertex 70. A special air-tight liquid cell was used (International Crystal Laboratories) with a path length of 0.5 $\mathrm{mm}$ and two $\mathrm{KBr}$ crystals at back and front sides to make it transparent for IR light. Spectra were recorded from 400 to 7500 $\mathrm{cm}^{-1}$, with a KBr beam splitter, a DLaTGS D301 detector, and a midIR source. Different solvents were used as indicated in the main text.

TEM and EDX. A Tecnai 20 or a Talos F200X (both $200 \mathrm{keV}$ ) electron microscope was used. The latter TEM also has the functionality of EDX.

Inductively Coupled Plasma Optical Emission Spectrometry. The exact concentration of lead in $\mathrm{PbTe}$ NCs could be obtained with the 
PerkinElmer Optima 8300 Optical Emission Spectrometer. The preparation of the calibration curve between 0 and $1 \mathrm{ppm}$ is based on a multielement solution with known amounts of ion species. This range of 5 dilutions was inserted into ICP-OES first, in order to establish the calibration curve.

$\mathrm{Pb}$ could not be measured when incorporated in a NC and therefore needed to be dissolved with the use of aqua regia. The next step was to dilute the samples by addition of $5 \% \mathrm{HNO}_{3}$ solution resulting in a $\mathrm{Pb}$ concentration in the region between 0 and $1 \mathrm{ppm}$. The wavelength chosen for $\mathrm{Pb}$ is $283.306 \mathrm{~nm}$.

${ }^{1} \mathrm{H}$ NMR measurements were performed using Agilent MRF400 equipped with a OneNMR probe and Optima Tune system. Spectra were recorded according to the following parameters: $400 \mathrm{MHz}$, $\mathrm{CDCl}_{3} 25{ }^{\circ} \mathrm{C}$. The ligand density of PbTe NCs was measured via a slightly modified method. ${ }^{40,42}$ In short, a suspension of a known PbTe NC concentration (measured via FT-IR) of an approximate concentration of approximately $4 \times 10^{-4} \mathrm{M}$ was measured including $10 \mu \mathrm{L}(0.05 \mathrm{M})$ ferrocene stock solution as the internal standard. The concentration of ligands was determined relatively to the internal standard, by integration of the ferrocene peak $(4.14 \mathrm{ppm})$ and the vinyl peak $(5.35 \mathrm{ppm})$, while normalizing for the amount of $\mathrm{H}$ atoms present (10:2). Using the size and concentration (FT-IR), we could determine the oleate density/NC. Measurements of $\mathrm{PbTe}$ NCs were performed using a longer relaxation delay $(30 \mathrm{~s})$ to allow complete relaxation. For $\mathrm{Pb}$ (oleate) ${ }_{2} \Delta=5.3(\mathrm{~m}, 4 \mathrm{H}, \mathrm{HC}=\mathrm{CH}), 2.2(\mathrm{t}, 4 \mathrm{H}$, $\left.{ }^{3} J_{\mathrm{HH}}=7.3 \mathrm{~Hz}, \mathrm{CH}_{2}\right), 2.0\left(\mathrm{~m}, 8 \mathrm{H}, \mathrm{CH}_{2}\right), 1.6\left(\mathrm{p}, 4 \mathrm{H},{ }^{3} \mathrm{~J}_{\mathrm{HH}}=7.8 \mathrm{~Hz}\right.$, $\left.\mathrm{CH}_{2}\right)$, 1.4-1.1 (m, $\left.42 \mathrm{H}, \mathrm{CH}_{2}\right)$, and $0.87\left(\mathrm{t}, 6 \mathrm{H},{ }^{3} \mathrm{~J}_{\mathrm{HH}}=6.7 \mathrm{~Hz}, \mathrm{CH}_{3}\right)$.

\section{ASSOCIATED CONTENT}

\section{S Supporting Information}

The Supporting Information is available free of charge on the ACS Publications website at DOI: 10.1021/acs.chemmater.8b05050.

Table of the measured atomic composition, FT-IR spectra of the oleate coordination of different NC sizes and in different solvents, deconvolution of the carboxylic vibrations and the corresponding table, and NMR spectra of NCs dissolved in different solvents (PDF)

\section{AUTHOR INFORMATION}

\section{Corresponding Author}

*E-mail: d.vanmaekelbergh@uu.nl.

ORCID $\odot$

Joep L. Peters: 0000-0002-3415-648X

Daniël Vanmaekelbergh: 0000-0002-3535-8366

\section{Author Contributions}

The manuscript was written through contributions of all the authors. All the authors have given approval to the final version of the manuscript.

\section{Notes}

The authors declare no competing financial interest.

\section{ACKNOWLEDGMENTS}

We want to thank Joen Hermans for the fruitful discussion. D.V. acknowledges support from NOW physics (FOM program 152) and from the ERC grant, grant 692691-FIRST STEP.

\section{ABBREVIATIONS}

NC, nanocrystal; EDX, energy-dispersive X-ray spectroscopy; TEM, transmission electron microscopy; XPS, X-ray photoelectron spectroscopy; ${ }^{1} \mathrm{H} \mathrm{NMR}$, proton-nucleair-magnetische resonantie; FT-IR, Fourier transform infrared spectroscopy;
ICP-OES, inductive coupled plasma optical emission spectrometry

\section{REFERENCES}

(1) Pietryga, J. M.; Park, Y.-S.; Lim, J.; Fidler, A. F.; Bae, W. K.; Brovelli, S.; Klimov, V. I. Spectroscopic and Device Aspects of Nanocrystal Quantum Dots. Chem. Rev. 2016, 116, 10513-10622.

(2) Urban, J. J.; Talapin, D. V.; Shevchenko, E. V.; Murray, C. B. Self-Assembly of PbTe Quantum Dots into Nanocrystal Superlattices and Glassy Films. J. Am. Chem. Soc. 2006, 128, 3248-3255.

(3) Hanrath, T. Colloidal nanocrystal quantum dot assemblies as artificial solids. J. Vac. Sci. Technol. A Vacuum, Surfaces, Film. 2012, 30, 030802.

(4) Alimoradi Jazi, M.; Janssen, V. A. E. C.; Evers, W. H.; Tadjine, A.; Delerue, C.; Siebbeles, L. D. A.; van der Zant, H. S. J.; Houtepen, A. J.; Vanmaekelbergh, D. Transport Properties of a Two-Dimensional $\mathrm{PbSe}$ Square Superstructure in an Electrolyte-Gated Transistor. Nano Lett. 2017, 17, 5238-5243.

(5) Xia, C.; Meeldijk, J. D.; Gerritsen, H. C.; de Mello Donega, C. Highly Luminescent Water-Dispersible NIR-Emitting Wurtzite CuInS2/ZnS Core/Shell Colloidal Quantum Dots. Chem. Mater. 2017, 29, 4940-4951.

(6) Koole, R.; Van Schooneveld, M. M.; Hilhorst, J.; Castermans, K.; David, P.; Strijkers, G. J.; Donegá, C. D. M.; Vanmaekelbergh, D.; Griffioen, W.; Nicolay, K.; Fayad, Z. A.; Meijerink, A.; Willem, J. M. Paramagnetic Lipid-Coated Silica Nanoparticles with a Fluorescent Quantum Dot Core: A New Contrast Agent Platform for Multimodality Imaging. Bioconjugate Chem. 2012, 19, 2471-2479.

(7) Mulder, W. J. M.; Koole, R.; Brandwijk, R. J.; Storm, G.; Chin, P. T. K.; Strijkers, G. J. Quantum Dots with a Paramagnetic Coating as a Bimodal Molecular Imaging Probe. Nano Lett. 2006, 6, 1-6.

(8) Deng, Z.; Jeong, K. S.; Guyot-Sionnest, P. Colloidal Quantum Dots Intraband Photodetectors. ACS Nano 2014, 8, 11707-11714.

(9) McDonald, S. A.; Konstantatos, G.; Zhang, S.; Cyr, P. W.; Klem, E. J. D.; Levina, L.; Sargent, E. H. Solution-Processed PbS Quantum Dot Infrared Photodetectors and Photovoltaics. Nat. Mater. 2005, 4, 138-142.

(10) Allan, G.; Delerue, C. Role of Impact Ionization in Multiple Exciton Generation in PbSe Nanocrystals. Phys. Rev. B: Condens. Matter Mater. Phys. 2006, 73, 205423.

(11) Ma, W.; Luther, J. M.; Zheng, H.; Wu, Y.; Alivisatos, A. P. Photovoltaic Devices Employing Ternary PbSxSe1-xNanocrystals. Nano Lett. 2009, 9, 1699-1703.

(12) Geuchies, J. J.; van Overbeek, C.; Evers, W. H.; Goris, B.; de Backer, A.; Gantapara, A. P.; Rabouw, F. T.; Hilhorst, J.; Peters, J. L.; Konovalov, O.; Petukhov, A. V.; Dijkstra, M.; Siebbeles, L. D. A.; van Aert, S.; Bals, S.; Vanmaekelbergh, D. In Situ Study of the Formation Mechanism of Two-Dimensional Superlattices from PbSe Nanocrystals. Nat. Mater. 2016, 15, 1248-1254.

(13) van Overbeek, C.; Peters, J. L.; van Rossum, S. A. P.; Smits, M.; van Huis, M. A.; Vanmaekelbergh, D. Interfacial Self-Assembly and Oriented Attachment in the Family of $\mathrm{PbX}(\mathrm{X}=\mathrm{S}, \mathrm{Se}, \mathrm{Te})$ Nanocrystals. J. Phys. Chem. C 2018, 122, 12464-12473.

(14) Peters, J. L.; Altantzis, T.; Lobato, I.; Jazi, M. A.; van Overbeek, C.; Bals, S.; Vanmaekelbergh, D.; Sinai, S. B. Mono- and Multilayer Silicene-Type Honeycomb Lattices by Oriented Attachment of PbSe Nanocrystals: Synthesis, Structural Characterization, and Analysis of the Disorder. Chem. Mater. 2018, 30, 4831-4837.

(15) Boneschanscher, M. P.; Evers, W. H.; Geuchies, J. J.; Altantzis, T.; Goris, B.; Rabouw, F. T.; van Rossum, S. A. P.; van der Zant, H. S. J.; Siebbeles, L. D. A.; Van Tendeloo, G.; Swart, I.; Hilhorst, J.; Petukhov, A. V.; Bals, S.; Vanmaekelbergh, D. Long-Range Orientation and Atomic Attachment of Nanocrystals in 2D Honeycomb Superlattices. Science 2014, 344, 1377-1380.

(16) Evers, W. H.; Goris, B.; Bals, S.; Casavola, M.; de Graaf, J.; van Roij, R.; Dijkstra, M.; Vanmaekelbergh, D. Low-Dimensional Semiconductor Superlattices Formed by Geometric Control over Nanocrystal Attachment. Nano Lett. 2012, 13, 2317-2323. 
(17) Kagan, C. R.; Murray, C. B. Charge Transport in Strongly Coupled Quantum Dot Solids. Nat. Nanotechnol. 2015, 10, 10131026.

(18) Žutić, I.; Fabian, J.; Das Sarma, S. Spintronics: Fundamentals and Applications. Rev. Mod. Phys. 2004, 76, 323.

(19) Beugeling, W.; Kalesaki, E.; Niquet, Y.; Vanmaekelbergh, D.; Delerue, C.; Smith, C. M. Topological States in Multi-Orbital HgTe Honeycomb Lattices. Nat. Commun. 2015, 6, 6316.

(20) Ibáñez, M.; Zamani, R.; Gorsse, S.; Fan, J.; Ortega, S.; Cadavid, D.; Morante, J. R.; Arbiol, J.; Cabot, A. Core-Shell Nanoparticles As Building Blocks for the Bottom-Up Production of Functional Nanocomposites: PbTe-PbS Thermoelectric Properties. ACS Nano 2013, 7, 2573-2586.

(21) Rowe, D. M. CRC Handbook of Thermoelectrics; CRC Press LLC: Boca Raton, FL, 1995.

(22) Harman, T. C.; Taylor, P. J.; Walsh, M. P.; Laforge, B. E. Quantum Dot Superlattice Thermoelectric Materials and Devices. Science 2002, 297, 2229-2232.

(23) Heremans, J. P.; Jovovic, V.; Toberer, E. S.; Saramat, A.; Kurosaki, K.; Charoenphakdee, A.; Yamanaka, S.; Snyder, G. J. Enhancement of Thermoelectric Efficiency in PbTe by Distortion of the Electronic Density of States. Science 2008, 321, 554-557.

(24) Faleev, S. V.; Léonard, F. Theory of Enhancement of Thermoelectric Properties of Materials with Nanoinclusions. Phys. Rev. B: Condens. Matter Mater. Phys. 2008, 77, 214304.

(25) Kanatzidis, M. G. Nanostructured Thermoelectrics: The New Paradigm? Chem. Mater. 2010, 22, 648-659.

(26) Piveteau, L.; Ong, T.-C.; Rossini, A. J.; Emsley, L.; Copéret, C.; Kovalenko, M. V. Structure of Colloidal Quantum Dots from Dynamic Nuclear Polarization Surface Enhanced NMR Spectroscopy. J. Am. Chem. Soc. 2015, 137, 13964-13971.

(27) Murphy, J. E.; Beard, M. C.; Norman, A. G.; Ahrenkiel, S. P.; Johnson, J. C.; Yu, P.; Mićić, O. I.; Ellingson, R. J.; Nozik, A. J. PbTe Colloidal Nanocrystals: Synthesis, Characterization, and Multiple Exciton Generation. J. Am. Chem. Soc. 2006, 128, 3241-3247.

(28) Ekuma, C. E.; Singh, D. J.; Moreno, J.; Jarrell, M. Optical Properties of $\mathrm{PbTe}$ and $\mathrm{PbSe}$. Phys. Rev. B: Condens. Matter Mater. Phys. 2012, 85, 085205.

(29) Moreels, I.; Lambert, K.; De Muynck, D.; Vanhaecke, F.; Poelman, D.; Martins, J. C.; Allan, G.; Hens, Z. Composition and Size-Dependent Extinction Coefficient of Colloidal PbSe Quantum Dots. Chem. Mater. 2007, 19, 6101-6106.

(30) Moreels, I.; Lambert, K.; Smeets, D.; De Muynck, D.; Nollet, T.; Martins, J. C.; Vanhaecke, F.; Vantomme, A. Size-Dependent Optical Properties of Colloidal PbS Quantum Dots. ACS Nano 2009, 3, 3023-3030.

(31) Allan, G.; Delerue, C. Confinement Effects in PbSe Quantum Wells and Nanocrystals. Phys. Rev. B: Condens. Matter Mater. Phys. 2004, 70, 245321.

(32) Tudury, G. E.; Marquezini, M. V.; Ferreira, L. G.; Barbosa, L. C.; Cesar, C. L. Effect of Band Anisotropy on Electronic Structure of $\mathrm{PbS}, \mathrm{PbSe}$, and $\mathrm{PbTe}$ Quantum Dots. Phys. Rev. B: Condens. Matter Mater. Phys. 2000, 62, 7357-7364.

(33) Hens, Z.; Moreels, I. Light Absorption by Colloidal Semiconductor Quantum Dots. J. Mater. Chem. 2012, 22, 10406.

(34) Xia, C.; Wu, W.; Yu, T.; Xie, X.; van Oversteeg, C.; Gerritsen, H. C.; de Mello Donega, C. Size-Dependent Band-Gap and Molar Absorption Coefficients of Colloidal CuInS2 Quantum Dots. ACS Nano 2018, 12, 8350-8361.

(35) Garnett, J. C. M. Colours in Metal Glasses and in Metallic Films. Philos. Trans. R. Soc. London, Ser. A 1904, 203, 385-420.

(36) Maryott, A. A.; Smith, E. R. Table of Dielectric Constants of Pure Liquids; United States Department of Commerce, 1951.

(37) Svane, A.; Christensen, N. E.; Cardona, M.; Chantis, A. N.; Schilfgaarde, M. Van; Kotani, T. Quasiparticle Self-Consistent GW Calculations for $\mathrm{PbS}, \mathrm{PbSe}$, and $\mathrm{PbTe}$ : Band Structure and Pressure Coefficients. Phys. Rev. B: Condens. Matter Mater. Phys. 2010, 81, 245120 .
(38) Suzuki, N.; Adachi, S. Optical Properties of PbTe. Jpn. J. Appl. Phys. 1994, 33, 193-198.

(39) Peters, J. L.; van den Bos, K. H. W.; Van Aert, S.; Goris, B.; Bals, S.; Vanmaekelbergh, D. Ligand-Induced Shape Transformation of PbSe Nanocrystals. Chem. Mater. 2017, 29, 4122-4128.

(40) Moreels, I.; Fritzinger, B.; Martins, J. C.; Hens, Z. Surface Chemistry of Colloidal PbSe Nanocrystals. J. Am. Chem. Soc. 2008, 130, 15081-15086.

(41) Choi, J. J.; Bealing, C. R.; Bian, K.; Hughes, K. J.; Zhang, W.; Smilgies, D.-M.; Hennig, R. G.; Engstrom, J. R.; Hanrath, T. Controlling Nanocrystal Superlattice Symmetry and Shape-Anisotropic Interactions through Variable Ligand Surface Coverage. J. Am. Chem. Soc. 2011, 133, 3131-3138.

(42) Anderson, N. C.; Hendricks, M. P.; Choi, J. J.; Owen, J. S. Ligand Exchange and the Stoichiometry of Metal Chalcogenide Nanocrystals: Spectroscopic Observation of Facile Metal-Carboxylate Displacement and Binding. J. Am. Chem. Soc. 2013, 135, 1853618548.

(43) Cass, L. C.; Malicki, M.; Weiss, E. A. The Chemical Environments of Oleate Species within Samples of Oleate-Coated PbS Quantum Dots. Anal. Chem. 2013, 85, 6974-6979.

(44) Mesubi, M. A. An Infrared Study of Zinc, Cadmium, and Lead Salts of Some Fatty Acids. J. Mol. Struct. 1982, 81, 61-71.

(45) Deacon, G.; Philiips, R. J. Relationships between the CarbonOxygen Stretching Frequencies of Carboxylato Complexes and the Type of Carboxylate Coordination. Coord. Chem. Rev. 1980, 33, 227250.

(46) Zelenák, V.; Vargová, Z.; Györyová, K. Correlation of infrared spectra of zinc(II) carboxylates with their structures. Spectrochim. Acta A Mol. Biomol. Spectrosc. 2007, 66, 262-72.

(47) Martínez-Casado, F. J.; Ramos-Riesco, M.; Rodríguez-Cheda, J. A.; Redondo-Yélamos, M. I.; Garrido, L.; Fernández-Martínez, A.; García-Barriocanal, J.; da Silva, I.; Durán-Olivencia, M.; Poulain, A. Lead(II) Soaps: Crystal Structures, Polymorphism, and Solid and Liquid Mesophases. Phys. Chem. Chem. Phys. 2017, 19, 17009-17018.

(48) Ellis, H. A.; White, N. A.; Hassan, I.; Ahmad, R. A room temperature structure for anhydrous lead (II) decanoate. J. Mol. Struct. 2002, 642, 71-76.

(49) Catalano, J.; Murphy, A.; Yao, Y.; Yap, G. P. A.; Zumbulyadis, N.; Centeno, S. A.; Dybowski, C. Coordination geometry of lead carboxylates - spectroscopic and crystallographic evidence. Dalton Trans. 2015, 44, 2340-2347.

(50) Strong, L.; Whitesides, G. M. Structures of Self-Assembled Monolayer Films of Organosulfur Compounds Adsorbed on Gold Single Crystals: Electron Diffraction Studies. Langmuir 1988, 4, 546558 .

(51) Ulman, A. Formation and Structure of Self-Assembled Monolayers. Chem. Rev. 1996, 96, 1533-1554.

(52) Maroncelli, M.; Qi, S. P.; Strauss, H. L.; Snyder, R. G. Nonplanar Conformers and the Phase Behavior of Solid N-Alkanes. J. Am. Chem. Soc. 1982, 104, 6237-6247.

(53) Snyder, R. G. Vibrational Study of the Chain Conformation of the Liquid n-Paraffins and Molten Polyethylene. J. Chem. Phys. 1967, 47, $1316-1360$.

(54) Barman, S.; Vasudevan, S. Contrasting Melting Behavior of Zinc Stearate and Zinc Oleate. J. Phys. Chem. Lett. B 2006, 110, 651654.

(55) Hostetler, M. J.; Stokes, J. J.; Murray, R. W.; Hill, C.; Carolina, $\mathrm{N}$. Infrared Spectroscopy of Three-Dimensional Self-Assembled Monolayers: N-Alkanethiolate Monolayers on Gold Cluster Compounds. Langmuir 1996, 12, 3604-3612.

(56) Badia, A.; Gao, W.; Singh, S.; Demers, L.; Cuccia, L.; Reven, L. Structure and Chain Dynamics of Alkanethiol-Capped Gold Colloids. Langmuir 1996, 12, 1262-1269.

(57) Badia, A.; Cuccia, L.; Demers, L.; Morin, F.; Lennox, R. B. Structure and Dynamics in Alkanethiolate Monolayers Self-Assembled on Gold Nanoparticles: A DSC, FT-IR, and Deuterium NMR Study. J. Am. Chem. Soc. 1997, 119, 2682-2692. 
(58) Hostetler, M. J.; Green, S. J.; Stokes, J. J.; Murray, R. W. Monolayers in Three Dimensions: Synthesis and Electrochemistry of $\omega$-Functionalized Alkanethiolate-Stabilized Gold Cluster Compounds. J. Am. Chem. Soc. 1996, 118, 4212-4213.

(59) Pradeep, T.; Mitra, S.; Nair, A. S.; Mukhopadhyay, R. Dynamics of Alkyl Chains in Monolayer-Protected Au and Ag Clusters and Silver Thiolates: A Comprehensive Quasielastic Neutron Scattering Investigation. J. Phys. Chem. B 2004, 108, 7012-7020.

(60) Mukhopadhyay, R.; Mitra, S.; Johnson, M.; Kumar, V. R. R.; Pradeep, T. Evolution of the Alkyl-Chain Dynamics in MonolayerProtected Gold Clusters. Phys. Rev. B: Condens. Matter Mater. Phys. 2007, 75, 075414.

(61) De Roo, J.; Yazdani, N.; Drijvers, E.; Lauria, A.; Maes, J.; Owen, J. S.; Van Driessche, I.; Niederberger, M.; Wood, V.; Martins, J. C.; Infante, I.; Hens, Z. Probing Solvent-Ligand Interactions in Colloidal Nanocrystals by the NMR Line Broadening. Chem. Mater. 2018, 30, 5485-5492.

(62) Wei, J.; Schaeffer, N.; Pileni, M.-P. Solvent-Mediated Crystallization of Nanocrystal 3D Assemblies of Silver Nanocrystals: Unexpected Superlattice Ripening. Chem. Mater. 2015, 28, 293-302.

(63) Yin, Y.; Alivisatos, A. P. Colloidal nanocrystal synthesis and the organic-inorganic interface. Nature 2005, 437, 664-670.

(64) Yang, Y.; Qin, H.; Jiang, M.; Lin, L.; Fu, T.; Dai, X.; Zhang, Z.; Niu, Y.; Cao, H.; Jin, Y.; Zhao, F.; Peng, X. Entropic Ligands for Nanocrystals: From Unexpected Solution Properties to Outstanding Processability. Nano Lett. 2016, 16, 2133-2138.

(65) Yang, Y.; Qin, H.; Peng, X. Intramolecular Entropy and SizeDependent Solution Properties of Nanocrystal - Ligands Complexes. 2016, 2131, 2-7. DOI: 10.1021/acs.nanolett.6b00737

(66) Goubet, N.; Richardi, J.; Albouy, P.-A.; Pileni, M.-P. Which Forces Control Supracrystal Nucleation in Organic Media? Adv. Funct. Mater. 2011, 21, 2693-2704.

(67) Quan, Z.; Xu, H.; Wang, C.; Wen, X.; Wang, Y.; Zhu, J.; Li, R.; Sheehan, C. J.; Wang, Z.; Smilgies, D.-M.; Luo, Z.; Fang, J. SolventMediated Self-Assembly of Nanocube Superlattices. J. Am. Chem. Soc. 2014, 136, 1352-1359.

(68) Wang, Z.; Bian, K.; Nagaoka, Y.; Fan, H.; Cao, Y. C. Regulating Multiple Variables to Understand the Nucleation and Growth and Transformation of PbS Nanocrystal Superlattices. J. Am. Chem. Soc. 2017, 139, 14476-14482.

(69) Hendricks, M. P.; Campos, M. P.; Cleveland, G. T.; Jen-La Plante, I.; Owen, J. S. A Tunable Library of Substituted Thiourea Precursors to Metal Sulfide Nanocrystals. Science 2015, 348, 12261230.

(70) Campos, M. P.; Hendricks, M. P.; Beecher, A. N.; Walravens, W.; Swain, R. A.; Cleveland, G. T.; Hens, Z.; Sfeir, M. Y.; Owen, J. S. A Library of Selenourea Precursors to PbSe Nanocrystals with Size Distributions near the Homogeneous Limit. J. Am. Chem. Soc. 2017, 139, 2296-2305. 